\title{
NORMAL OPERATORS AND UNIFORMLY ELLIPTIC SELF-ADJOINT PARTIAL DIFFERENTIAL EQUATIONS(1)
}

\author{
BY \\ LEO SARIO AND GEORGES WEILL
}

1. Let $C_{H}^{1}(V)$ be the class of functions with Hölder-continuous first derivatives in a region $V$ of Euclidean $n$-space $R^{n}$. Let $a_{t k} \in C_{H}^{1}(V), i, k=1, \cdots, n$, with $a_{i k}=a_{k i}$, such that

$$
\frac{1}{\lambda} \sum_{i} \xi_{i}^{2}<a_{i k}(x) \xi_{i} \xi_{k}<\lambda \sum_{i} \xi_{i}^{2}
$$

in $V$ for some constant $\lambda>0$ and all reals $\xi_{i}$; here Einstein's summation convention is adopted. Consider the self-adjoint uniformly elliptic partial differential equation

$$
E u \equiv \frac{\partial}{\partial x_{k}}\left(a_{i k}(x) \frac{\partial u}{\partial x_{i}}\right)=0 .
$$

We shall present a method of finding solutions with given singularities and given behavior near the boundary of $V$. Extremal properties of such solutions, to be called principal solutions, will be established.

A more detailed description of the problem and its significance will be given in No. 6 below, after the necessary concepts have been introduced.

Our approach is based on the linear operator method previously used for the Laplacian [6], [7]. The generalization, while parallel, reveals delicate differences. The existence of the $L_{0}$-operator, one of our basic tools, for every regular subregion is trivial in the case of Riemann surfaces; in the present general context it is a deep result established by G. Fichera on pp. 195-202 of [4]. The convergence of the principal functions of exhausting subregions offered difficulties not overcome in the present paper, and extremal properties are given for regular subregions only.

For further extensions and applications of our method we refer to [8] and [9]. In a new direction (not containing earlier work) the problem was given an elegant formulation in [1].

\section{$\S 1$. Preliminaries.}

2. We shall call a subregion $\Omega$ of $V \subset R^{n} H$ ölder-bordered if

Presented to the Society, April 21, 1964 under the title Normal linear operators and some self-adjoint partial differential equations; received by the editors August 24, 1964.

(1) The work was sponsored by the U.S. Army Research Office (Durham), Grant DAARO(D) 31-124-G499, University of California, Los Angeles, and the National Science Foundation, Grant NSF-G-19751, Yale University. The authors are indebted to Professors Lipman Bers and Felix Browder for helpful discussions. 
(a) $\partial \Omega$ is compact in $V$,

(b) every $x \in \partial \Omega$ has a neighborhood $N_{x}$ and a homeomorphism $h$ of $N_{x}$ with the unit ball $B \subset R^{n}$ such that $h\left(N_{x} \cap \partial \Omega\right)$ is the intersection of $B$ with a coordinate plane $P, h\left(N_{x} \cap \Omega\right)$ is one of the half-balls constituting $B \backslash P$, and $h^{-1}$ is in $C_{H}^{1}(B)$.

A Hölder-bordered region shall be called Hölder-regular, or simply regular, if

(c) $\overline{\mathbf{\Omega}}$ is compact in $V$,

(d) each component of $V-\Omega$ is noncompact in $V$.

3. At a point $x$ of the boundary $\partial \Omega$ of a Hölder-bordered region we denote by $\partial / \partial n$ the normal derivative in the Euclidean metric, directed toward the exterior of $\Omega$ unless specified otherwise. We set

$$
a=\left[\sum_{i}\left(\sum_{k} a_{i k} \cos \left(x_{k}, n\right)\right)^{2}\right]^{1 / 2}
$$

and define the conormal derivative $\partial / \partial v$ by

$$
a \frac{\partial}{\partial v}=a_{i k} \cos \left(x_{k}, n\right) \frac{\partial}{\partial x_{i}} .
$$

We shall call $\int_{\partial \Omega} a(\partial u / \partial v) d S$ the $f u x$ of $u$ across the border of a Hölder-bordered $\Omega, u$ being in $C_{H}^{1}$ in an open set containing $\partial \Omega$.

Solutions of (2) in $C_{H}^{1}$ will be referred to simply as "solutions". By a "solution in a set $E$ " will be meant the restriction to $E$ of a solution in an open set containing $E$. For a regular $\Omega$ and solutions $u, v$ in $\bar{\Omega}$ the Green's formula reads [5]

$$
\int_{\partial \Omega} a\left(v \frac{\partial u}{\partial v}-u \frac{\partial v}{\partial v}\right) d S=0
$$

where $d S$ is the surface element of $\partial \Omega$. As a consequence, the mean value property, the Harnack inequality, and the maximum principle are valid [3].

The norm over $V$ is defined as $M(u)=M(u, u)$, where

$$
M(u, v)=\int_{V} a_{i k} \frac{\partial u}{\partial x_{i}} \frac{\partial v}{\partial x_{k}} d V
$$

The triangle inequality

$$
M^{1 / 2}(u+v) \leqq M^{1 / 2}(u)+M^{1 / 2}(v)
$$

is proved in the usual manner.

4. Let $V_{0}$ be a compact set in $V \subset R^{n}$. We consider a family $\{u\}$ of solutions in $V$ with sgn $u \mid V_{0} \neq$ const.

LEMMA. There exists a positive constant $q<1$, independent of $u$, such that

$$
\max _{V_{0}}|u| \leqq q \sup _{V}|u|
$$


Proof. The lemma is trivial if $\sup _{V}|u|=0$ or $\infty$. In other cases we normalize by $\sup _{V}|u|=1$. Suppose there did not exist any constant $q<1$ with $\max _{V_{0}}|u| \leqq q$. Then there would exist a sequence $\left\{u_{n}\right\}$ with $\max _{V_{0}}\left|u_{n}\right| \rightarrow 1$. The points $\left\{z_{n}\right\}$ in $V_{0}$ where the maxima are taken have a subsequence, again denoted by $\left\{z_{n}\right\}$, tending to $z_{0} \in V_{0}$, say. The coefficients $a_{i k}$ being continuous in the compact set $V_{0}$, the set $\left\{u_{n}\right\}$ of uniformly bounded solutions is compact $[2$, p. 344]. A subsequence tends to a solution $u_{0}$ with $\left|u_{0}\left(z_{0}\right)\right|=1, \operatorname{sgn} u_{0} \mid V_{0} \neq$ const. This violates the maximum principle.

\section{$\S 2$. The problem.}

5. Let $V_{1}$ be the complement in $V$ of the closure of a regular region, and let $F$ be the family of solutions $f$ on $\alpha_{1}=\partial V_{1}$.

DEFINITION. L is a normal operator if for $f \in F$ the function $L f$ is a solution in $\bar{\nabla}_{1}$ with the following properties:

$$
\begin{gathered}
L f \mid \alpha_{1}=f, \\
L\left(c_{1} f_{1}+c_{2} f_{2}\right)=c_{1} L f_{1}+c_{2} L f_{2}, \\
\min f \leqq L f \leqq \max f, \\
\int_{\alpha_{1}} a \frac{\partial L f}{\partial v} d S=0 .
\end{gathered}
$$

The existence of operators $L$ will be established in $\S \S 4-5$.

6. Let $s$ be a solution in $\bar{V}_{1}$. We shall construct a solution $p$ in $V$ that imitates the behavior of $s$ in $\bar{V}_{1}$. More precisely, for a given $s$ and $L$ we require that $p \mid \bar{V}_{1}=s+L(p-s)$. This means that $p \mid \bar{V}_{1}$ deviates from $s$ by a bounded solution, and we shall give explicit bounds for the deviation. Moreover, $p-s$ enjoys boundary properties determined by the choice of $L$.

$V_{1}$ need not be connected. For instance, given a region $V^{*} \subset R^{n}$ and points $z_{i} \in V^{*}, i=1, \cdots, m$, we may choose $V$ as $V^{*} \backslash \bigcup\left\{z_{i}\right\}$. Given disjoint neighborhoods $N_{i}$ of the $z_{i}, V_{1}$ can be taken as the union of the punctured neighborhoods $V_{1 i}=N_{i} \mid z_{\imath}$ and of a neighborhood $V_{1 \beta}=V_{1}^{*}$ of the ideal boundary $\beta$ of $V^{*}$ with $\bar{V}_{1 \beta} \cap\left\{\bigcup \bar{V}_{1 i}\right\}=\varnothing$.

In $V_{1 i}, s$ may have a singularity at $z_{i}$, and $L$ may be the operator giving the solution of the Dirichlet problem in $N_{i}$. In $V_{1 \beta}, s$ can have an arbitrary growth when approaching $\beta$, and $L$ can be given separately in each component of $V_{1 \beta}$. Thus we are dealing with the problem of constructing solutions with given singularities and prescribed boundary behavior.

The problem is given further interest by the fact that certain operators $L$ give significant extremum properties to the corresponding $p$. We shall call $p$ the principal solution for given $s, L$. 
7. Once $s$ and $L$ have been prescribed, the principal solution is unique up to an additive constant. Indeed, for two such functions $p^{\prime}, p^{\prime \prime}$ we have

$$
\max _{V-V_{1}}\left(p^{\prime}-p^{\prime \prime}\right)=\max _{\alpha_{1}}\left(p^{\prime}-p^{\prime \prime}\right)
$$

by the maximum principle, and

$$
\max _{\bar{V}_{1}}\left(p^{\prime}-p^{\prime \prime}\right)=\max _{\alpha_{1}}\left(p^{\prime}-p^{\prime \prime}\right)
$$

by (11). Consequently $\max _{V}\left(p^{\prime}-p^{\prime \prime}\right)$ is taken on $\alpha_{1}$, and $p^{\prime}-p^{\prime \prime}$ must reduce to a constant.

Without loss of generality we assume that $s \mid \alpha_{1}=0$, for otherwise we can replace $s$ by $s-L s$.

The function $p$ is constant if and only if $s \equiv 0$. Sufficiency is seen from $p=L p$ and the maximum principle. Necessity is obvious.

For the existence of $p$ it is necessary that the flux of $s$ vanish,

$$
\int_{\alpha_{1}} a \frac{\partial s}{\partial v} d S=0 .
$$

This follows directly from $\int_{\alpha_{1}} a(\partial p / \partial v) d S=0$ and (12). The essence of the following main existence theorem is that (13) is also sufficient.

\section{§3. Main existence theorem.}

8. Let $V$ be a region in $R^{n}$, and $V_{1}$ the complement in $V$ of a regular subregion of $V$, with border $\alpha_{1}$. Let $L$ be a normal operator (9)-(12) for $V_{1}$, and $s$ with $s \mid \alpha_{1}=0$ a solution in $\bar{V}_{1}$ of the elliptic equation (2) whose coefficients satisfy the conditions stated in No. 1. We shall write $L p$ for $L\left(p \mid \alpha_{1}\right)$.

THEOREM. The vanishing of the flux (13) of $s$ is necessary and sufficient for the existence of a principal solution $p$ on $V$ of (2), characterized by

$$
p \mid \bar{V}_{1}=s+L p \text {. }
$$

The function is unique up to an additive constant and it reduces to a constant if and only if $s \equiv 0$.

Proof. Let $V_{0}$ be a regular subregion of $V$ with border $\alpha_{0}=\partial V_{0} \subset V_{1}, \alpha_{1} \subset V_{0}$. Let $L^{\prime}$ be the operator that for functions on $\alpha_{0}$ gives solutions of the Dirichlet problem in $\bar{V}_{0}$. It suffices to find $p \mid \alpha_{0}$, for then

$$
p\left|\bar{V}_{0}=L^{\prime} p, \quad p\right| \bar{V}_{1}=s+L p,
$$

where again $L^{\prime} p$ stands or $L^{\prime}\left(p \mid \alpha_{0}\right)$. We set $K=L L^{\prime}$ and seek $p \mid \alpha_{0}$ with 


$$
p=s+K p
$$

on $\alpha_{0}$.

9. We shall show that

$$
p=\sum_{0}^{\infty} K^{n} s
$$

converges uniformly on $\alpha_{0}$. Then $K$ can be applied term by term, for

$$
\left|K \sum_{0}^{\infty} K^{n} s-\sum_{1}^{m+1} K^{n} s\right|=\left|K \sum_{m+1}^{\infty} K^{n} s\right| \leqq\left|\sum_{m+1}^{\infty} K^{n} s\right|
$$

which tends to 0 as $m \rightarrow \infty$. Consequently $K p=\sum_{1}^{\infty} K^{n_{s}}=p-s$ as desired.

Let $\omega \in C^{1}$ in $\bar{V}_{0} \cap \bar{V}_{1}$ satisfying (2) in $V_{0} \cap V_{1}$ with $\omega\left|\alpha_{0}=0, \omega\right| \alpha_{1}=1$. For a solution $\sigma$ in $\bar{V}_{0} \cap \bar{V}_{1}$ with $\int_{\alpha_{0}} a(\partial \sigma / \partial v) d S=0$ we have by (5)

$$
\int_{\alpha_{0}} \sigma a \frac{\partial \omega}{\partial \nu} d S=\int_{\alpha_{1}} \sigma a \frac{\partial \omega}{\partial \nu} d S .
$$

This holds, in particular, for $\sigma=s, L^{\prime} \phi, L \psi, K \phi$, where $\phi, \psi$ are restrictions to $\alpha_{0}, \alpha_{1}$ of solutions in open sets containing $\alpha_{0}, \alpha_{1}$, respectively.

We claim that

$$
\int_{\alpha_{1}} K^{n} s a \frac{\partial \omega}{\partial v} d S=0
$$

for $n \geqq 0$. By assumption this is true for $n=0$. Suppose it holds for $n=i$. Then by (18) the integral vanishes over $\alpha_{0}$ as well:

$$
\int_{\alpha_{0}} L^{\prime} K^{i} s a \frac{\partial \omega}{\partial v} d S=0 .
$$

Again $\alpha_{0}$ can be replaced by $\alpha_{1}$, and subsequently $L^{\prime}$ by $L L^{\prime}=K$. The statement follows.

From $\cos (n, v)>0[5]$ and the maximum principle we have $\partial \omega / \partial v>0$. This together with $a>0$ gives $\operatorname{sgn} K^{n} s \mid \alpha_{1} \neq$ const. Lemma 4 applies:

$$
|K s|\left|\alpha_{0} \leqq q \max _{\alpha_{0}}\right| s|, \quad| K^{n} s|| \alpha_{0} \leqq q^{n} \max _{\alpha_{0}}|s|,
$$

whence the uniform convergence of (17). On setting

$$
m=\min _{\alpha_{0}} s, \quad M=\max _{\alpha_{0}} s,
$$

one can also easily see (cf. [7]) that

$$
q^{n} m \leqq K^{n} s \mid \alpha_{0} \leqq q^{n} M .
$$


10. We have actually proved more than Theorem 8 states:

THEOREM. The principal solution is given by the Neumann series $p \mid \alpha_{0}=$ $\Sigma_{0}^{\infty} K^{n}$ s and satisfies the inequalities

$$
\begin{aligned}
& \frac{m}{1-q} \leqq p \mid \bar{V}_{0} \leqq \frac{M}{1-q}, \\
& \frac{m}{1-q} \leqq p-s \mid \bar{V}_{1} \leqq \frac{M}{1-q} .
\end{aligned}
$$

Indeed, (21) gives these bounds for $p \mid \alpha_{0}$, and by the maximum principle they hold for $p \mid \bar{V}_{0}$, hence for $p\left|\alpha_{1}, p-s\right| \alpha_{1}$, and $p-s \mid \bar{V}_{1}$.

\section{$\S 4$. Normal operators for regular regions.}

11. In this section $V_{1}$ stands for a regular region in $R^{n}$ with disconnected border partitioned into disjoint sets $\alpha_{1}$ and $\beta$ of components. Let $f$ be the restriction to $\alpha_{1}$ of a solution in an open set containing $\alpha_{1}$. Denote by $E\left(\bar{V}_{1}\right)$ the family of solutions in $\bar{V}_{1}$ and set

$$
U=\left\{u\left|u \in E\left(\bar{V}_{1}\right), u\right| \alpha_{1}=f\right\} .
$$

In this class we single out the functions $u_{0}$ and $u_{1}$ determined by the conditions

$$
\begin{gathered}
\frac{\partial u_{0}}{\partial v} \mid \beta=0 \\
u_{1} \mid \beta=c \text { (const.), } \quad \int_{\alpha_{1}} a \frac{\partial u_{1}}{\partial v} d S=0 .
\end{gathered}
$$

The existence of $u_{0}$ has been demonstrated by G. Fichera [4, pp. 195-202]. The flux of $u_{1}$ can be taken across $\beta$, where it is negative for $c=\min f$, positive for $c=\max f$, and increases monotonically with $c$. This ensures the existence of the constant $c$ with property (26).

The functions $u_{0}$ and $u_{1}$ are uniquely determined and are related to $f$ by linear operators:

$$
u_{0}=L_{0} f, \quad u_{1}=L_{1} f .
$$

It is readily seen that $L_{0}, L_{1}$ are normal in the sense of No. 5 .

12. For $\lambda \in R$ set

$$
u_{\lambda}=(1-\lambda) u_{0}+\lambda u_{1}=L_{\lambda} f \text {, }
$$

where the operator $L_{\lambda}$ is again normal. We shall derive an extremal property of $u_{\lambda}$ in the class

$$
U_{0}=\left\{u \mid u \in U, \int_{\alpha_{1}} a \frac{\partial u}{\partial v} d S=0\right\}
$$


This property will serve to establish the existence of normal operators for arbitrary Hölder-bordered regions $V_{1}$.

For $u, v \in U_{0}$ set

$$
A(u, v)=\int_{\alpha_{1}} u a \frac{\partial v}{\partial v} d S, \quad B(u, v)=\int_{\beta} u a \frac{\partial v}{\partial v} d S,
$$

where $\partial / \partial v$ is taken toward the interior of $V_{1}$ on $\alpha_{1}$, exterior to it on $\beta$. Write

$$
A(u)=A(u, u), \quad B(u)=B(u, u),
$$

and let $M(u)=M(u, u)$ stand for the norm (6) over $V_{1}$.

LEMma. On a regular $\bar{V}_{1} \subset R^{n}$, the function $u_{0}$ minimizes $M(u)$, and $u_{1}$ minimizes $A(u)+B(u)$, in $U_{0}$.

We shall prove, more generally, that

$$
B(u)+(2 \lambda-1) A(u)=\lambda^{2} A\left(u_{1}\right)-(1-\lambda)^{2} A\left(u_{0}\right)+M\left(u-u_{\lambda}\right) .
$$

Thus $u_{\lambda}$ minimizes the functional on the left and the minimum is given by the first two terms on the right. We also have an explicit expression for the deviation from this minimum: $M\left(u-u_{\lambda}\right)$.

Proof. Clearly $A\left(u-u_{\lambda}\right)=B\left(u, u_{0}\right)=B\left(u_{1}, u\right)=0$ for $u \in U_{0}$. In the decomposition

$$
M\left(u-u_{\lambda}\right)=B(u)+B\left(u_{\lambda}\right)-B\left(u, u_{\lambda}\right)-B\left(u_{\lambda}, u\right)
$$

we rewrite the last three terms:

$$
\begin{aligned}
B\left(u_{\lambda}\right) & =\lambda(1-\lambda) B\left(u_{0}, u_{1}\right) \\
& =\lambda(1-\lambda)\left(B\left(u_{0}, u_{1}\right)-B\left(u_{1}, u_{0}\right)\right) \\
& =\lambda(1-\lambda)\left(A\left(u_{1}\right)-A\left(u_{0}\right)\right), \\
B\left(u, u_{\lambda}\right) & =\lambda\left(B\left(u, u_{1}\right)-B\left(u_{1}, u\right)\right) \\
& =\lambda\left(A\left(u_{1}\right)-A(u)\right), \\
B\left(u_{\lambda}, u\right) & =(1-\lambda)\left(B\left(u_{0}, u\right)-B\left(u, u_{0}\right)\right) \\
& =(1-\lambda)\left(A(u)-A\left(u_{0}\right)\right) .
\end{aligned}
$$

Here the transfers from $\beta$ to $\alpha_{1}$ are justified by Green's formula. The lemma follows.

\section{§5. Operators for noncompact regions.}

13. Now let $V_{1}$ be an arbitrary Hölder-bordered region in $R^{n}$ with border $\alpha_{1}=\partial V_{1}$ and let $\Omega \subset V_{1}$ be a regular subregion with border $\partial \Omega=\alpha_{1} \cup \beta_{\Omega}, \beta_{\Omega} \subset V_{1}$. Denote by $u_{\Omega \lambda}$ the function $u_{\lambda}$ of No. 12 constructed for $\Omega$. The family $\left\{u_{\Omega \lambda}\right\}$ of uniformly bounded functions is normal (cf. No. 4): for every nested exhausting 
sequence of the $\Omega$ 's there is a subsequence $\left\{\Omega_{n}\right\}$ such that the corresponding functions $u_{n}=u_{\Omega_{n} \lambda}$ converge to a limiting solution $u_{\lambda}$ in $V_{1}$. The convergence is uniform on compact subsets of $V_{1}$, and hence on those of $\bar{V}_{1}$, and $u_{\lambda}$ belongs to the class $U_{0}$ defined by (24), (29) for the noncompact $\bar{V}_{1}$.

We set

$$
\begin{aligned}
B_{\Omega}(u, v) & =\int_{\beta_{\Omega}} u a \frac{\partial v}{\partial v} d S, \\
B(u) & =\lim _{\Omega \rightarrow V_{1}} B_{\Omega}(u),
\end{aligned}
$$

where $B_{\Omega}(u)$ stands for $B_{\Omega}(u, u)$; we consider functions $u$ with a finite $B(u)$. For $B_{\Omega_{n}}(u)$ we write $B_{n}(u)$ and set

$$
F_{n}(u)=B_{n}(u)+(2 \lambda-1) A(u) .
$$

LEMMA. Every limiting function $u_{\lambda}=\lim _{n \rightarrow \infty} u_{n}$ minimizes the functional

$$
F(u)=B(u)+(2 \lambda-1) A(u)
$$

in $U_{0}$ and the value of the minimum is

$$
F\left(u_{\lambda}\right)=\lim _{n \rightarrow \infty} F_{n}\left(u_{n}\right) .
$$

Proof. We have

$$
F\left(u_{\lambda}\right)=\lim _{m \rightarrow \infty} F_{m}\left(u_{\lambda}\right)=\lim _{m \rightarrow \infty} \lim _{n \rightarrow \infty} F_{m}\left(u_{n}\right) .
$$

Moreover, $F_{m}\left(u_{n}\right) \leqq F_{n}\left(u_{n}\right)$ for $m \leqq n$, and consequently

On the other hand

$$
F\left(u_{\lambda}\right) \leqq \liminf _{n \rightarrow \infty} F_{n}\left(u_{n}\right)
$$

and

$$
F_{n}\left(u_{n}\right) \leqq F_{n}(u) \leqq F(u)
$$

$$
\limsup _{n \rightarrow \infty} F_{n}\left(u_{n}\right) \leqq \inf _{U_{0}} F(u) \leqq F\left(u_{\lambda}\right)
$$

We conclude that

$$
\min _{U_{0}} F(u)=F\left(u_{\lambda}\right)=\lim _{n \rightarrow \infty} F_{n}\left(u_{n}\right) .
$$

14. The norm of $u \in U_{0}$ over $\bar{V}_{1}$ is

$$
M(u)=B(u)-A(u) .
$$

THEOREM. On an arbitrary Hölder-bordered $\bar{V}_{1} \subset R^{n}$ there are unique functions $u_{0}$ and $u_{1}$ that minimize in $U_{0}$ the functionals $M(u)$ and $A(u)+B(u)$, respectively. 
We shall again derive the more general result

$$
B(u)+(2 \lambda-1) A(u)=\lambda^{2} A\left(u_{1}\right)-(1-\lambda)^{2} A\left(u_{0}\right)+M\left(u-u_{\lambda}\right) .
$$

Proof. For any limiting function $u_{\lambda}=\lim u_{n}$ and for $u \in U_{0}$ set $h=u-u_{\lambda}$. Then for $\varepsilon \in R$,

$$
F_{n}\left(u_{\lambda}+\varepsilon h\right)=F_{n}\left(u_{\lambda}\right)+\varepsilon^{2} M_{n}(h)+\varepsilon I_{n},
$$

where

$$
I_{n}=B_{n}\left(u_{\lambda}, h\right)+B_{n}\left(h, u_{\lambda}\right)+(2 \lambda-1) A\left(u_{\lambda}, h\right) .
$$

If $M(h)<\infty$, the first three terms in (40) have limits as $n \rightarrow \infty$ and a fortiori $I_{n}$ tends to a limit $I$ :

$$
F\left(u_{\lambda}+\varepsilon h\right)=F\left(u_{\lambda}\right)+\varepsilon^{2} M(h)+\varepsilon I .
$$

By the minimum property of $u_{\lambda}, d F / d \varepsilon=0$ for $\varepsilon=0$ and we infer that $I=0$. For $\varepsilon=1$ this gives

$$
F(u)=F\left(u_{\lambda}\right)+M\left(u-u_{\lambda}\right),
$$

which by (37) is the desired deviation formula (39).

That two minimizing functions are identical follows from

$$
M\left(u^{\prime}-u^{\prime \prime}\right)=F\left(u^{\prime}\right)-F\left(u^{\prime \prime}\right)=0 .
$$

The uniqueness enables us to introduce the operators $L_{0}, L_{1}$ for the arbitrary Hölder-bordered $V_{1}: u_{\lambda}=L_{\lambda} f$. Due to the uniform convergence of $u_{n}$, the operators are normal.

\section{§6. Extremal properties of principal solutions.}

15. In this section $V \subset R^{n}$ is a regular region with border $\beta,\left(A_{i k}\right)$ signifies the adjoint matrix of $\left(a_{i k}\right)$, and $A$ is its determinant. Designate by

$$
d(x, y)=\left[A_{i k}(y)\left(x_{i}-y_{i}\right)\left(x_{k}-y_{k}\right)\right]^{1 / 2}
$$

the "elliptic distance" between $x, y \in V$ and set

$$
\sigma(x, y)=\frac{(d(x, y))^{2-n}}{(n-2) \omega_{n} \sqrt{A(y)}},
$$

where $\omega_{n}$ is the area of the unit $n$-sphere. (In the case $n=2,(42)$ is replaced by the corresponding logarithmic singularity.)

Let $C_{a}, C_{b} \subset V$ be balls with disjoint closures, centered at $a, b$. The Green's functions of (2) in $\bar{C}_{a}, \bar{C}_{b}$ with singularities at $a, b$ can be written (up to sign)

$$
\begin{aligned}
& s(x, a)=\sigma(x, a)+h(x), \\
& s(x, b)=-\sigma(x, b)+k(x),
\end{aligned}
$$


where for some $\kappa>0$ and all $i, j=1, \cdots, n$ the function $h(x)$ has the properties

$$
h(x)=O\left(r^{x+2-n}\right), \quad \frac{\partial h(x)}{\partial x_{i}}=O\left(r^{\kappa+1-n}\right), \quad \frac{\partial^{2} h(x)}{\partial x_{i} \partial x_{k}}=O\left(r^{\kappa-n}\right)
$$

in terms of the Euclidean distance $r=|x-a|$, and $k(x)$ satisfies analogous conditions near $b$. By definition, $s(x, a)\left|\partial C_{a}=s(x, b)\right| \partial C_{b}=0$. The flux of $s(x, a)$ across $\partial C_{a}$ is -1 and that of $s(x, b)$ across $\partial C_{b}$ is 1 .

16. For given $\lambda, \mu \in R$ let $P_{\mu+\lambda}$ be the class of solutions in $\bar{V}-a-b$ with singularities

$$
\begin{aligned}
& p \mid \bar{C}_{a}=(\lambda+\mu) s(x, a)+e(x), \\
& p \mid \bar{C}_{b}=-(\lambda+\mu) s(x, b)+f(x),
\end{aligned}
$$

where $e$ and $f$ are solutions of (2) in $\bar{C}_{a}$ and $\bar{C}_{b}$ respectively, $f(b)=0$. The flux of $p$ across $\partial C_{a}$ is $-(\lambda+\mu)$ and that across $\partial C_{b}$ is $\lambda+\mu$. Let $p_{i} \in P_{1}$ be the principal solution determined by $L_{i}(i=0,1)$ and set

$$
\begin{gathered}
p_{\mu \lambda}=\mu p_{0}+\lambda p_{1} \in P_{\mu+\lambda}, \\
B(p)=\int_{\beta} p a \frac{\partial p}{\partial v} d S .
\end{gathered}
$$

Denote by $h_{i}$ the function $h$ corresponding to $p_{i}$.

THEOREM. The functions $p_{0}$ and $p_{1}$ minimize the functionals $B(p)-e(a)$ and $B(p)+e(a)$, respectively, in $P_{1}$.

More generally, $p_{\mu \lambda}$ has in $P_{\mu+\lambda}$ the minimum property

$$
B(p)+(\lambda-\mu) e(a)=\lambda^{2} h_{1}(a)-\mu^{2} h_{0}(a)+M\left(p-p_{\mu \lambda}\right) .
$$

Proof. We again start with $M\left(p-p_{\mu \lambda}\right)=B(p)+B\left(p_{\mu \lambda}\right)-B\left(p, p_{\mu \lambda}\right)-B\left(p_{\mu \lambda}, p\right)$. In analogy with (30) let $A_{a}, A_{b}$ stand for integrals taken over $\partial C_{a}$ and $\partial C_{b}$. Then $B\left(p_{\mu \lambda}\right)$ is the sum of

$$
\mu \lambda\left[A_{a}\left(s+h_{0}, s+h_{1}\right)-A_{a}\left(s+h_{1}, s+h_{0}\right)\right]
$$

and a similar expression in terms of $A_{b}$. Since $s \mid \partial C_{a}=0$, and by Green's formula $A_{a}\left(h_{0}, h_{1}\right)-A_{a}\left(h_{1}, h_{0}\right)=0$, the only nonvanishing terms are the mean values $A_{a}\left(h_{i}, s\right)=-h_{i}(a)$ (e.g. Miranda [5, formulas (6.5), (7.5), and (9.3)]). By virtue of the normalization $k(b)=0$ the contribution of the $A_{b}$-terms vanishes and we obtain

$$
B\left(p_{\mu \lambda}\right)=\mu \lambda\left(h_{1}(a)-h_{0}(a)\right) .
$$

Similarly

$$
\begin{aligned}
& B\left(p, p_{\mu \lambda}\right)=\lambda\left[(\mu+\lambda) h_{1}(a)-e(a)\right], \\
& B\left(p_{\mu \lambda}, p\right)=\mu\left[e(a)-(\mu+\lambda) h_{0}(a)\right],
\end{aligned}
$$


and the theorem follows.

17. If $\mu+\lambda=0$ the competing class $P_{0}$ consists of regular solutions $u$ in $V$ with $u(b)=0$.

Corollary. The difference $p_{0}-p_{1}$ minimizes in $P_{0}$ the functional $M(u)$ $-2 u(a)$. The value of the minimum is $h_{1}(a)-h_{0}(a)$ and the deviation from the minimum is $M\left(u-p_{0}+p_{1}\right)$.

Using the special case $u=0$ one obtains an explicit expression for the norm of $p_{0}-p_{1}$ :

$$
M\left(p_{0}-p_{1}\right)=h_{0}(a)-h_{1}(a) .
$$

18. For solutions with finite norm we obtain a bound for $u(a)$ :

COROLlary. The inequality

$$
(u(a))^{2} \leqq M(u)\left(h_{0}(a)-h_{1}(a)\right)
$$

holds for all regular solutions $u \in P_{0}$.

Proof. Replace $u$ by $c u, c=$ const., to obtain

$$
c^{2} M(u)-2 c u(a)=h_{1}(a)-h_{0}(a)+M\left(c u-p_{0}+p_{1}\right) .
$$

Since the last term is nonnegative the desired result follows on substituting $c=u(a) / M(u)$.

\section{REFERENCES}

1. F. Browder, Principal functions for elliptic systems of differential equations, Bull. Amer. Math. Soc. 71 (1965), 342-344.

2. R. Courant and D. Hilbert, Methods of mathematical physics, Vol. II, Interscience, New York, 1962.

3. W. Feller, Uber die Lösungen der linearen partiellen Differentialgleichungen zweiter Ordnung vom elliptischen Typus, Math. Ann. 102 (1930), 633-649.

4. G. Fichera, Alcuni recenti sviluppi della teoria dei problemi al contorno per le equazioni alle derivate parziali lineari, Convegno Internazionale sulle Equazioni Lineari alle Derivate Parziali, Trieste, 1954, pp. 174-227, Edizioni Cremonese, Rome, 1955.

5. C. Miranda, Equazioni alle derivate parziali di tipo ellittico, Springer, Berlin, 1955.

6. L. Sario, A linear operator method on arbitrary Riemann surfaces, Trans. Amer. Math. Soc. 72 (1952), 281-295.

7. —_, Classification of locally Euclidean spaces, Nagoya Math. J. 25 (1965), 87-111.

8. L. Sario and N. Fukuda, Harmonic functions with given values and minimum norms in Riemannian spaces, Proc. Nat. Acad. Sci. U.S.A. 53 (1965), $270-273$.

9. L. Sario, M. Schiffer and M. Glasner, The span and principal functions in Riemannian spaces, J. Analyse Math. 15 (1965), 115-134.

UNIVERSITY OF CALIFORNIA, Los ANgeles, California

YALE UNIVERSITY, New Haven, ConNecticut 